Flávia Bellesia Souzedo

OChttps://orcid.org/0000-0002-5520-6474

Lisiane Bizarro ${ }^{1}$

Ohttps:/orid.org/0000-0003-3070-5944

Ana Paula Almeida de Pereira² Ohttps://orcid. org/0000-0002-3779-6976

\section{O eixo intestino-cérebro e sintomas depressivos: uma revisão sistemática dos ensaios clínicos randomizados com probióticos}

\author{
The gut-brain axis and depressive symptoms: a systematic \\ review of randomized clinical trials with probiotics \\ D0I: 10.1590/0047-2085000000285
}

\section{RESUMO}

Objetivo: Reconhece-se atualmente a relevância do eixo intestino-cérebro para a compreensão de comportamentos e doenças mentais ou psiquiátricas. O presente estudo teve por objetivo analisar os efeitos do consumo de probióticos sobre sintomas depressivos e depressão maior. Métodos: $O$ presente estudo constitui uma revisão de ensaios clínicos randomizados duplos-cegos ou triplos-cegos, placebo-controlados, publicados entre 2010 e 2020. Foi realizada busca por artigos nas bases de dados PubMed, ScienceDirect e Google Scholar. Resultados: Oito artigos compuseram a amostra do presente estudo. Os resultados entre estudos são controversos e indicam que a relação de causalidade entre o consumo de probióticos e o alívio de sintomas depressivos ainda não foi estabelecida. Conclusões: Mais ensaios clínicos randomizados duplos-cegos ou triplos-cegos, placebo-controlados, que controlem potenciais fatores de confusão (p. ex.: dieta, uso de antibióticos), são necessários para verificar consistentemente a relação causal entre o consumo de probióticos e o alívio de sintomas depressivos.

\section{PALAVRAS-CHAVE}

Eixo intestino-cérebro, sintomas depressivos, probióticos, ensaios clínicos randomizados.

\section{ABSTRACT}

Objective: Currently, the gut-brain axis has been highlighted as an important aspect for understanding behavior and mental illnesses. The present study aimed to analyze the effects of probiotic consumption on depressive symptoms and major depression. Methods: A review of randomized, double or triple blind and placebo-controlled trials, published between 2010 and 2020, was performed in PubMed, ScienceDirect and Google Scholar. Results: Eight articles were selected to compose the sample. Findings between studies are controversial, and indicate that a causal relation between probiotic consumption and relieve of depressive symptoms is not yet established. Conclusion: More randomized, double or triple blind, placebo-controlled trials are necessary to consistently verify the causal relation between probiotic consumption and relieve of depressive symptoms.

\section{KEYWORDS}

Gut-brain axis, depressive symptoms, probiotics, randomized controlled trials. 


\section{INTRODUÇÃO}

Estima-se que $10^{14}$ microrganismos residam no intestino de um adulto, quantia essa equivalente a dez vezes o número de células de todo o corpo humano ${ }^{1-3}$. A maior parte desses microrganismos são bactérias - denominadas "comensais" -, que, juntas, somam até 1.000 espécies distintas, e cujas quantidade e variedade pode variar devido a fatores como genética, idade, estresse, uso de fármacos e nutrição ${ }^{1,4-6}$. Esses pequenos organismos contêm sua própria carga genética, de modo que o intestino humano apresenta mais de 3 milhões de genes microbiais - número que ultrapassa em muito a quantia de genes humanos (20.000) e que, por isso, têm sido chamados de "metagenoma"1-3. Projetos de larga escala como o Projeto Microbioma Humano (http:// commonfund.nih.gov/hmp) dedicam-se ao estudo pormenorizado de tais microrganismos, com o objetivo de promover a saúde dos indivíduos?.

Atualmente, sabe-se que o eixo intestino-cérebro é constituído por rotas bidirecionais e, para comunicação, utiliza vias como o sistema nervoso parassimpático (em especial, o nervo vago), o sistema imune, o sistema neuroendócrino e o sistema circulatório - que permite a passagem de metabólitos e neurotransmissores produzidos pelo intestino 1,8-10. Recentemente, tornou-se evidente que a microbiota intestinal pode influenciar o funcionamento do eixo intestino-cérebro e alterar funções cerebrais e até mesmo o comportamento ${ }^{2,5,11}$. Essa descoberta se deu por diversos estudos realizados com: 1) animais (geralmente, camundongos) livres de germes ${ }^{12-15} ; 2$ ) animais tratados com probióticos, prebióticos ou antibióticos ${ }^{16-19}$; e 3) transplante fecal ${ }^{20-22}$.

Reconhece-se atualmente a relevância do eixo intestino-cérebro para a compreensão de comportamentos e doenças mentais ou psiquiátricas ${ }^{11,23,24}$. A microbiota intestinal é capaz de influenciar circuitos neurais e comportamentos associados com uma resposta estressora, e patologias como a depressão estão associadas a mudanças na microbiota ${ }^{2,11,25}$. A composição da microbiota intestinal de pessoas com depressão parece diferir da microbiota de indivíduos saudáveis ${ }^{26,27}$, apresentando menor diversidade na microbiota intestinal, bem como maiores níveis de marcadores inflamatórios. Pacientes com síndrome do intestino irritável (SII) e outras doenças inflamatórias do trato gastrointestinal costumam ter ansiedade e depressão como comorbidades ${ }^{28,29}$, possivelmente por desregulações no metabolismo do triptofano ${ }^{30}$. No estudo de Kurokawa et al. ${ }^{31}$, sujeitos com complicações intestinais (como a SII) receberam transplante fecal de pacientes saudáveis; após o transplante, verificou-se alívio de sintomas depressivos.

O estudo pioneiro de Sudo et al. ${ }^{32}$ reportou que ratos livres de germes, quando expostos a um evento estressor, mostraram hiperativação do eixo hipófise-pituitária-adrenal (HPA) e diminuição de fator neurotrófico derivado do cérebro (BNDF, do inglês brain-derived neurotrophic factor), neurotrofina fundamental para a neurogênese. Sujeitos deprimidos costumam apresentar alterações no eixo HPA ${ }^{33,34}$, e a neurogênese hipocampal parece relevante para a eficácia de antidepressivos e o controle do humor ${ }^{35}$. Posteriormente, os cientistas colonizaram os ratos com o probiótico Bifidobacterium infantis, o que estabilizou a atividade do eixo HPA. Estudos posteriores realizados com roedores indicaram que intervenções com probióticos foram capazes de atenuar comportamentos do tipo depressivo e ansioso $36-38$.

A depressão é uma psicopatologia de etiologia complexa. São características dessa psicopatologia sintomas como alterações no ciclo sono-vigília, alterações no apetite, alterações na libido, anedonia e humor triste ${ }^{39}$. A depressão envolve alterações em regiões cerebrais específicas, como o hipotálamo, o hipocampo, a amígdala e o córtex pré-frontal ${ }^{39}$. Algumas pessoas apresentam depressão subclínica, isto é, apresentam alguns sintomas dessa psicopatologia, porém não se encaixam por completo no diagnóstico de depressão maior (DM), o qual, de acordo com o Manual Diagnóstico e Estatístico de Transtornos Mentais - $5^{a}$ edição $(\mathrm{DSM}-5)^{40}$, é caracterizado pela presença de pelo menos cinco sintomas durante, ao menos, duas semanas.

De acordo com a Organização Mundial da Saúde (http:// www.who.int/news-room/fact-sheets/detail/depression), atualmente o quadro de DM afeta mais de 300 milhões de pessoas, sendo considerada a principal causa de problemas e incapacidade em todo o mundo. Em termos financeiros, são gastos bilhões de dólares anualmente para manejar essa condição. Apesar dos altos gastos, os resultados do tratamento (geralmente, realizado via fármacos) permanecem abaixo da expectativa: um em cada dois pacientes não responde bem ao tratamento, $40 \%$ daqueles que respondem têm recaídas e muitos pioram apesar do tratamento ${ }^{41}$. Tendo em vista os impactos na saúde pública, existe a necessidade de novas abordagens capazes de tratar ou prevenir a depressão. Intervenções na microbiota intestinal (por exemplo: o consumo de probióticos) podem ser uma via alternativa de tratamento e, possivelmente, de prevenção dessa psicopatologia ${ }^{42-44}$. O presente estudo teve por objetivo compreender os impactos do uso de probióticos sobre sintomas depressivos, incluindo a DM, de acordo com ensaios clínicos randomizados duplos-cegos ou triplos-cegos.

\section{MÉTODOS}

O presente estudo constitui uma revisão de ensaios clínicos randomizados duplos-cegos ou triplos-cegos, publicados entre 2010 e 2020, que tiveram o objetivo de verificar o impacto do uso de probióticos sobre sintomas de depressão. A busca por artigos foi realizada nas bases de dados PubMed, ScienceDirect e Google Scholar utilizando as seguintes 
palavras-chave: "Gut brain axis AND probiotics AND depress* AND randomized". A busca foi realizada pela primeira autora (F.B.S.), com base em título e resumo, e registrada em documento Word contendo informações como data da busca, palavras-chave utilizadas, número total de artigos encontrados, número de resumos selecionados, total de artigos avaliados para elegibilidade e total de artigos excluídos da amostra final com os motivos para a exclusão. Para os artigos incluídos na amostra final, gerou-se uma planilha contendo informações detalhadas a respeito de cada estudo. As autoras L.B.A. e A.P.A.P. revisaram e aprovaram o procedimento metodológico.

Foram considerados critérios de inclusão: ensaios clínicos randomizados duplos-cegos ou triplos-cegos realizados com seres humanos de ambos os sexos; população de jovens adultos ou adultos; com o foco em sintomas depressivos ou DM; publicações em língua inglesa ou portuguesa. Foram considerados critérios de exclusão: artigos que utilizaram outra metodologia que não ensaios clínicos randomizados duplos-cegos ou triplos-cegos; realizados com outros seres que não humanos (por exemplo: roedores) ou com humanos de apenas um dos sexos; em população de crianças, adolescentes ou idosos; com foco em outra psicopatologia que não a depressão; publicados em outras línguas que não o inglês ou português.

\section{RESULTADOS}

A figura 1 contém o fluxograma de seleção dos estudos. Um total de 587 artigos foi encontrado nas bases de dados ScienceDirect, PubMed e Google Scholar, e oito artigos foram incluídos na amostra final: Akkasheh et al. ${ }^{45}$, Steenbergen et al. ${ }^{46}$, Pinto-Sanchez et al. ${ }^{47}$, Romijn et al. ${ }^{48}$, Kazemi et al. ${ }^{49}$, Majeed et al. ${ }^{50}$, Chahwan et al. ${ }^{51}$ e Rudzki et al.52 (ver tabela 1 para descrição detalhada de cada artigo).

Os oito ensaios clínicos randomizados, duplos-cegos ou triplos-cegos, foram realizados em diferentes regiões geográficas O objetivo de tais estudos foi, de maneira geral, investigar o impacto do consumo de probióticos sobre sintomas de depressão. A maior parte dos estudos realizou intervenções com sujeitos deprimidos e que não consumiam antidepressivos ${ }^{45-48,50,51}$, avaliou sintomas depressivos via instrumentos diversos ${ }^{45-52}$ e utilizou uma combinação de espécies bacterianas ${ }^{45,46,48-51}$. Em todos os estudos, os sujeitos foram aleatoriamente alocados ao grupo placebo ou experimental e receberam a intervenção durante períodos que variaram de quatro ${ }^{46}$ a doze semanas ${ }^{50}$. Alterações significativas em sintomas depressivos foram avaliadas comparando os escores (médias) do grupo experimental e placebo no início e ao final das intervenções.

Foram identificadas diferenças metodológicas entre estudos concernentes à forma de seleção e avaliação de participantes, critérios de exclusão e inclusão, controle de potenciais fatores de confusão, entre outros aspectos. Os resultados dos estudos são conflitantes. Alguns encontraram que a suplementação com probióticos foi útil para aliviar sintomas depressivos ${ }^{45,46,49,50}$, enquanto outros reportaram não encontrar diferenças significativas para sintomas depressivos entre o grupo experimental e o grupo controle após a intervenção ${ }^{48,51,52}$.
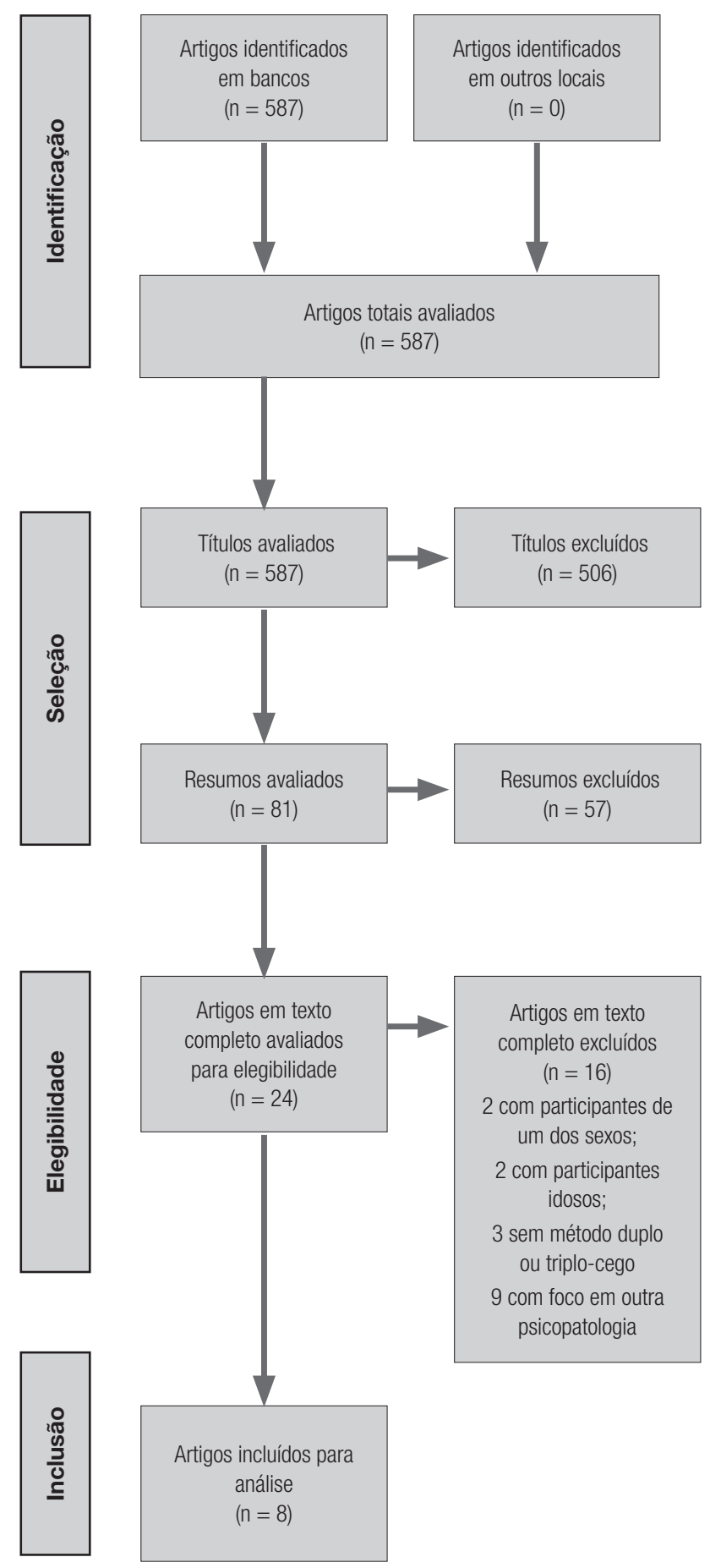

Figura 1. Fluxograma de seleção de artigos nas bases de dados. 
Tabela 1. Ensaios clínicos randomizados, duplos-cegos ou triplos-cegos, placebo controlados

\begin{tabular}{|c|c|c|c|c|c|}
\hline Autor, ano & Objetivo & Método & $\begin{array}{l}\text { Principais resultados/ } \\
\text { Conclusões }\end{array}$ & Limitações & Bactérias utilizadas \\
\hline $\begin{array}{l}\text { Akkasheh et al., } \\
\text { 2015, Irã }\end{array}$ & $\begin{array}{l}\text { Determinar os efeitos da } \\
\text { ingestão de probióticos em } \\
\text { sintomas de depressão e o } \\
\text { status metabólico de } \\
\text { pacientes com depressão } \\
\text { maior. }\end{array}$ & $\begin{array}{l}\text { Estudo duplo-cego; } 40 \\
\text { pacientes adultos com } \\
\text { depressão maior foram } \\
\text { aleatoriamente alocados } \\
\text { em grupos para receber } \\
\text { suplementos probióticos } \\
\text { ou placebo durante } 8 \\
\text { semanas. }\end{array}$ & $\begin{array}{l}\text { Depois da intervenção } \\
\text { com probióticos, os } \\
\text { pacientes tiveram } \\
\text { significativo decréscimo } \\
\text { nos índices do Inventário } \\
\text { de Depressão de Beck } \\
\text { (BDI) se comparados ao } \\
\text { grupo placebo. }\end{array}$ & $\begin{array}{l}\text { Não sabem quais bactérias } \\
\text { geraram os resultados } \\
\text { positivos. Participantes } \\
\text { tomavam antidepressivos. }\end{array}$ & $\begin{array}{l}\text { Lactobacillus acidophilus, } \\
\text { Lactobacillus casei e } \\
\text { Bifidobacterium bifidum }\end{array}$ \\
\hline $\begin{array}{l}\text { Steenbergen et al., } \\
2015, \text { Holanda }\end{array}$ & $\begin{array}{l}\text { Testar se probióticos de } \\
\text { espécies variadas podem } \\
\text { reduzir a reatividade } \\
\text { cognitiva em indivíduos } \\
\text { sem depressão. }\end{array}$ & $\begin{array}{l}\text { Estudo triplo-cego; } 20 \\
\text { participantes jovens sem } \\
\text { desordens de humor } \\
\text { receberam suplementos } \\
\text { probióticos por quatro } \\
\text { semanas, enquanto } 20 \\
\text { participantes controles } \\
\text { receberam placebo pelo } \\
\text { mesmo período. }\end{array}$ & $\begin{array}{l}\text { Comparado ao grupo } \\
\text { placebo, os participantes } \\
\text { que receberam os } \\
\text { probióticos apresentaram } \\
\text { redução significativa de } \\
\text { reatividade cognitiva ao } \\
\text { humor triste, incluindo } \\
\text { redução de ruminação e } \\
\text { pensamentos agressivos. }\end{array}$ & $\begin{array}{l}\text { Não controlou dieta e uso } \\
\text { de probióticos. Amostra } \\
\text { composta } \\
\text { predominantemente por } \\
\text { pessoas do sexo feminino. }\end{array}$ & $\begin{array}{l}\text { Bifidobacterium bifidum W23, } \\
\text { Bifidobacterium lactis W52, } \\
\text { Lactobacillus acidophilus W37, } \\
\text { Lactobacillus brevis W63, } \\
\text { L. casei W56, Lactobacillus } \\
\text { salivarius } \\
\text { W24 e Lactococcus lactis } \\
\text { (W19 e W58) }\end{array}$ \\
\hline
\end{tabular}

$\begin{array}{ll}\text { Romijn et al., } & \text { Investigar se probióticos } \\ \text { 2017, Nova } & \text { melhoram o humor, 0 } \\ \text { Zelândia } & \text { estresse e a ansiedade. }\end{array}$

Pinto-Sanchez et

al., 2017, Canadá

Avaliar os efeitos do probiótico Bifidobacterium longum NCC3001 (BL) na ansiedade e depressão de pacientes com síndrome do intestino irritável (SII).

\begin{tabular}{|c|c|}
\hline $\begin{array}{l}\text { Kazemi et al., } \\
\text { 2019, Irã }\end{array}$ & $\begin{array}{l}\text { Comparar o efeito da } \\
\text { suplementação com } \\
\text { probióticos e prebióticos } \\
\text { na pontuação do Inventário } \\
\text { de Depressão de Beck } \\
\text { (BDI) e nos níveis de } \\
\text { triptofano. }\end{array}$ \\
\hline Autor, ano & Objetivo \\
\hline \multirow[t]{2}{*}{$\begin{array}{l}\text { Majeed et al., } \\
\text { 2018, Índia }\end{array}$} & $\begin{array}{l}\text { Verificar a eficácia do } \\
\text { probiótico Bacillus }\end{array}$ \\
\hline & $\begin{array}{l}\text { coagulans MTCC } 5856 \\
\text { para depressão maior em } \\
\text { pacientes com síndrome } \\
\text { do intestino irritável. }\end{array}$ \\
\hline $\begin{array}{l}\text { Chahwan et al., } \\
\text { 2019, Austrália }\end{array}$ & $\begin{array}{l}\text { Determinar o efeito do } \\
\text { consumo de probióticos } \\
\text { em sintomas de depressão } \\
\text { em pacientes com } \\
\text { depressão moderada ou } \\
\text { severa. }\end{array}$ \\
\hline
\end{tabular}

Estudo duplo-cego; 79 participantes jovens com sintomas de depressão e/ ou ansiedade foram alocados aleatoriamente para receber probióticos ou placebo durante 8 semanas. Biomarcadores foram monitorados.

Estudo duplo-cego; 44 adultos com síndrome do intestino irritável e depressão moderada ou severa foram alocados aleatoriamente para receber probióticos ou placebo por seis semanas.

Estudo duplo-cego; 110 pacientes adultos com depressão foram aleatoriamente designados para receber probióticos (Lactobacillus helveticus e Bifidobacterium longum), prebióticos (galactooligossacarídeos) ou placebo por 8 semanas.

Método

Estudo duplo-cego; 40 participantes adultos deprimidos foram aleatoriamente designados para receber probióticos ou placebo por 90 dias.

Estudo triplo-cego; 71 participantes adultos com depressão foram aleatoriamente designados para receber probióticos ou placebo por oito semanas. Follow-up de um mês.
Não foram encontradas diferenças entre 0 grupo que recebeu probióticos e o grupo placebo.
Não controlou dieta. Não Lactobacillus helveticus e realizou análise microbial. Bifidobacterium longum

\section{Houve melhora}

significativa nos sintomas depressivos se comparado ao grupo placebo, mas não se manteve no follow-up de dez semanas.

A suplementação com probióticos resultou em diminuição significativa nos escores do BDI $e$ aumento significativo de triptofano, se comparado aos grupos controle e de prebióticos.
Período de intervenção

menor que de outros

estudos. Não controlou dieta.

\section{Bifidobacterium longum}

$\begin{array}{ll}\text { Participantes tomavam } & \text { Lactobacillus helveticus e } \\ \text { antidepressivos. } & \text { Bifidobacterium longum }\end{array}$

Principais resultados/ Conclusões

A suplementação com 0 probiótico gerou alívio dos sintomas depressivos.

Bifidobacterium longum

Todos os participantes (grupo experimental e placebo) tiveram redução de sintomas depressivos. 0 grupo experimental reportou menor reatividade cognitiva.
Testes específicos seriam necessários para análise de mudanças sutis da microbiota intestinal.
Bifidobacterium bifidum W23, Bifidobacterium lactis W51, Bifidobacterium lactis W52, L. acidophilus W37, Lactobacillus brevis W63, Lactobacillus casei W56, Lactobacillus salivarius W24, Lactococcus lactis W19 e Lactococcus lactis W58 


\begin{tabular}{|c|c|c|c|c|c|}
\hline Autor, ano & Objetivo & Método & $\begin{array}{c}\text { Principais resultados/ } \\
\text { Conclusões }\end{array}$ & Limitações & Bactérias utilizadas \\
\hline $\begin{array}{l}\text { Rudzki et al., } \\
\text { 2019, Polônia }\end{array}$ & $\begin{array}{l}\text { Verificar o efeito do } \\
\text { consumo do probiótico } \\
\text { Lactobacillus plantarum } \\
\text { 299v em afetos e } \\
\text { cognições de pacientes } \\
\text { com depressão maior. }\end{array}$ & $\begin{array}{l}\text { Estudo duplo-cego; } 79 \\
\text { participantes adultos que } \\
\text { tomavam antidepressivos } \\
\text { foram aleatoriamente } \\
\text { designados para receber } \\
\text { probióticos ou placebo por } \\
\text { oito semanas. } \\
\text { Biomarcadores analisados. }\end{array}$ & $\begin{array}{l}\text { Não foram observadas } \\
\text { mudanças significativas } \\
\text { para sintomas depressivos } \\
\text { entre os grupos. } \\
\text { Observaram-se melhoria } \\
\text { do desempenho cognitivo } \\
\text { e diminuição de } \\
\text { quinurenina. }\end{array}$ & $\begin{array}{l}\text { Participantes tomavam } \\
\text { antidepressivos. Alguns } \\
\text { testes cognitivos foram } \\
\text { realizados em } \\
\text { subamostras. Não } \\
\text { controlou dieta. }\end{array}$ & Lactobacillus Plantarum 299v \\
\hline
\end{tabular}

\section{DISCUSSÃO}

Nesta amostra de ensaios clínicos randomizados, duplos-cegos ou triplos-cegos e placebo-controlados, encontrou-se que os resultados dos estudos são conflitantes, de modo que alguns estudos relataram efeitos positivos de probióticos sobre sintomas depressivos ${ }^{45,46,49,50}$ e outros não encontraram diferenças significativas entre grupos após a intervenção ${ }^{48,51,52}$. Diferenças metodológicas podem ter contribuído para os diferentes resultados obtidos, a começar pelos instrumentos utilizados para a seleção dos participantes, que variam amplamente entre os estudos, de modo que alguns utilizaram instrumentos diversos ${ }^{45-47,51}$, outros, os critérios do Manual Diagnóstico e Estatístico de Transtornos Mentais - 4a edição DSM-IV45,50,51, e ainda um estudo ${ }^{49}$ selecionou sujeitos com base no consumo prévio de antidepressivos.

Para avaliar sintomas depressivos, a maior parte dos estudos utilizou mais de um instrumento ${ }^{46,47,50-52}$. Entre os instrumentos utilizados, encontram-se o Inventário de Depressão de Beck (BDI) $)^{45,46,49,51}$, a Escala Hamilton de Depressão (HAM-D) $)^{50,52}$ e a escala Montgomery-Asberg Depression Rating Scale (MADRS) ${ }^{48,49}$. Alguns estudos ${ }^{45,49,50,52}$ não mencionam pontos de corte para dividir grupos com sintomas depressivos leves, moderados ou graves, enquanto outros o fazem $^{46-48,51}$. Entre aqueles que utilizam as mesmas escalas e estabeleceram pontos de corte, observa-se que estes são distintos ${ }^{46,51}$. Três estudos avaliaram os sujeitos apenas no início e no fim das intervenções ${ }^{45,46,49}$; os demais avaliaram também durante o processo. Em todos os estudos, compararam-se os escores (médias) do grupo experimental e os do grupo placebo obtidos no início e no fim das intervenções, a fim de identificar alterações de escores significativas.

A HAM-D é uma escala preenchida pelo clínico, cuja versão mais utilizada contém 17 itens, e é considerada por alguns autores como "padrão-ouro" de referência para a construção de outras escalas, sendo amplamente usada em ensaios clínicos ${ }^{53}$. Entretanto, outros autores questionam o status de "padrão-ouro", sugerindo falhas psicométricas e conceituais relevantes ${ }^{54}$. A MADRS é uma escala composta por 10 itens, preenchidos pelo clínico ${ }^{48}$, e foi desenhada para ser mais sensível a mudanças de sintomas depressivos ${ }^{55}$. Trata-se, porém, de um instrumento mais breve e menos detalhado, que não possibilita acessar sintomas atípicos e que não inclui entrevista estruturada ${ }^{56}$. Já o BDI é um inventário de autorregistro, composto por 21 itens, sendo talvez o mais utilizado globalmente para avaliar depressão $0^{57,58}$. Contudo, não há consenso em relação aos pontos de corte a serem utilizados $^{57}$; e, diferente dos outros instrumentos, o BDI, especialmente em sua primeira versão, possui enfoque sobre atitudes cognitivas, e não sobre sintomas de depressão (por exemplo: alterações no apetite e sono) $)^{58}$.

É possível que essa variação nos instrumentos utilizados resulte em grupos de participantes com diferentes perfis sintomatológicos, uma vez que cada teste psicológico possui suas particularidades e porque não há consenso relativo aos pontos de corte ${ }^{53,58}$. Todos os instrumentos supracitados possuem vantagens e desvantagens, no entanto sugere-se que estudos futuros não apliquem unicamente o BDI, como fizeram alguns estudos desta amostra ${ }^{45,49}$, já que seu enfoque é sobre atitudes cognitivas. Além disso, devem explicitar os pontos de corte estabelecidos para os instrumentos utilizados, o que pode ajudar a esclarecer se os eventuais efeitos benéficos de probióticos se aplicam a diferentes graus de sintomas depressivos.

Existem variações importantes nos critérios de inclusão e exclusão utilizados pelos estudos, o que pode afetar consideravelmente o resultado final. A maior parte dos estudos incluiu sujeitos com sintomas depressivos, mas que não tomam antidepressivos, porém alguns incluíram sujeitos que tomavam antidepressivos ${ }^{49,52}$. Os critérios de exclusão são ainda mais variados. Alguns estudos deixam de lado critérios importantes como o uso de drogas ${ }^{48}$, o uso de medicações prescritas ${ }^{45}$, o consumo de probióticos antes das intervenções ${ }^{46,52}$ e gravidez e lactação ${ }^{46,47}$. Além disso, somente alguns inserem critérios de exclusão como cirurgias abdominais ${ }^{47,50}$, risco para suicídio $^{48}$ e dietas específicas ${ }^{49}$. Estudos futuros devem estar atentos ao delinear seus critérios de inclusão e exclusão, uma vez que podem levar a resultados enganosos se mal delimitados.

Poucos estudos controlaram fatores como dieta e atividade física, apesar de a literatura apontar para sua importância na composição da microbiota intestinal25,59,60 Especificamente, dois estudos ${ }^{45,49}$ controlam dieta e atividade física, e dois outros controlam somente dieta ${ }^{50,51}$. A principal forma de controle se deu via recordatórios fornecidos pelos participantes em diferentes etapas do estudo, porém alguns estudos utilizaram também questionários ${ }^{49,51}$. O controle de fatores com 
potencial impacto sobre a microbiota intestinal pode ajudar a obter resultados mais confiáveis a respeito da intervenção realizada. Estudos futuros devem controlar tais fatores, priorizando o uso de instrumentos sempre que possível, a fim de mensurar eventuais mudanças com maior precisão.

O período de intervenção variou de quatro ${ }^{46}$ a doze semanas ${ }^{50}$ entre os estudos, e a maior parte realizou a intervenção em oito semanas ${ }^{45,48,49,51}$. Diferentes gêneros e espécies de bactérias foram utilizados, sendo os gêneros mais frequentes Bifidobacterium e Lactobacillus. A maior parte dos estudos utilizou uma combinação de espécies bacterianas, e o número de espécies combinadas variou amplamente entre os estudos, de modo que alguns utilizaram duas ${ }^{48,49}$, três ${ }^{45}$ ou mais espécies ${ }^{46,51}$. Ainda, houve aqueles que utilizaram somente uma espécie $e^{47,50,52}$. O uso simultâneo de diferentes probióticos pode dificultar a compreensão de quais foram, especificamente, as bactérias que produziram o efeito benéfico e para qual nível (leve, moderado ou grave) de depressão. Estudos futuros devem intervir utilizando apenas um tipo de bactéria e realizar as análises microbiais necessárias para detectar alterações sutis.

Além disso, a maior parte dos estudos não relatou follow-up após a intervenção. Sugere-se que trabalhos futuros busquem avaliar em maior extensão temporal os efeitos do uso de probióticos sobre os sintomas de depressão, a fim de poder esclarecer se os benefícios advêm da suplementação ou de comportamentos novos, como sugerem Chahwan et al. ${ }^{51}$.

É interessante notar que Kazemi et al. ${ }^{49}$ e Romijn et al. ${ }^{48}$ usaram os mesmos probióticos (B. longum e L. helveticus), entretanto os resultados de ambos os estudos são divergentes: para Romijn et al. ${ }^{48}$, não houve diferenças entre os grupos controle e de intervenção, enquanto para Kazemi et al. ${ }^{49} \mathrm{O}$ uso de tais probióticos resultou em diminuição significativa nos escores do BDI. Ambos os estudos utilizaram a mesma quantidade de probióticos, realizaram a intervenção durante o mesmo período de tempo (oito semanas) e controlaram dados clínicos e demográficos dos participantes. Uma diferença importante entre os dois estudos é que, no estudo de Kazemi et al. ${ }^{49}$, os participantes fizeram suplementação de probióticos conjuntamente com a de prebióticos, o que pode ter influenciado o resultado, uma vez que o consumo deles pode alterar a composição e a atividade da microbiota intestinal, influenciando o bem-estar do hospedeiro61,62.

Além disso, no estudo de Kazemi et al. ${ }^{49}$, o uso de antidepressivos por três meses ou mais antes do início da intervenção foi um critério de inclusão, enquanto no estudo de Romijn et al. ${ }^{48}$ esse foi um critério de exclusão. Antidepressivos podem ter efeitos antimicrobiais relacionados à efetividade de tais drogas ${ }^{63}$. $O$ efeito antimicrobial de antidepressivos pode ser benéfico, ocasionando uma restauração do equilíbrio da microbiota intestinal, ou prejudicial em caso de uso crônico, levando ao empobrecimento da microbiota intestinal ${ }^{63}$, o que favorece a manifestação de sintomas depressivos ${ }^{64,65}$. Diferente de Kazemi et al. ${ }^{49}$, Rudzki et al. ${ }^{52}$ não explicitam o tempo de tratamento com antidepressivos. Em ambos os estudos, observa-se que a classe de antidepressivos mais frequente foram os inibidores seletivos de receptação de serotonina (ISRS).

É interessante notar que outras terapias (por exemplo: psicoterapias) não foram mencionadas em grande parte dos estudos, de maneira que somente Romijn et al. ${ }^{48}$ comentam a inclusão de indivíduos que frequentavam psicoterapia por mais de seis meses e sugerem que esse pode ter sido um fator de confusão. Estudos futuros devem controlar esse aspecto, para que os resultados não sejam enviesados. Em caso de inclusão de participantes que consomem antidepressivos, o tempo de tratamento deve ser mencionado.

Similarmente, os estudos de Steenbergen et al. ${ }^{46}$ e de Chahwan et al. ${ }^{51}$ utilizaram coquetéis de probióticos, compostos por bactérias semelhantes, e encontraram resultados divergentes: no estudo de Steenbergen et al. ${ }^{46}$, o uso de probióticos promoveu o alívio de sintomas depressivos; no estudo de Chahwan et al. ${ }^{51}$, ambos os participantes do grupo experimental e placebo tiveram redução de sintomas depressivos. Conforme discutido previamente, são muitos os aspectos divergentes entre estudos: as escalas utilizadas para a seleção e a avaliação dos participantes, bem como os pontos de corte estabelecidos; os critérios de inclusão e de exclusão; a ausência de controle sobre fatores que interagem com a microbiota intestinal; as diferenças na extensão das intervenções, nas espécies bacterianas utilizadas. Conjuntamente, todos esses aspectos podem ter colaborado para os resultados contrastantes e tornam a comparação entre os estudos quase inviável, dada a heterogeneidade das amostras.

A literatura sugere que a maior parte das intervenções com probióticos para alívio de sintomas depressivos ocorre com indivíduos sem diagnóstico de depressão ${ }^{16}$, no entanto isso não ocorreu nesta amostra. A maior parte dos estudos realizou intervenções com indivíduos deprimidos, exceto o de Steenbergen et al. ${ }^{46}$. Intervenções com indivíduos deprimidos podem ser mais adequadas se quisermos compreender a relação causal entre o uso de probióticos e o alívio de sintomas depressivos. Futuros estudos devem estar atentos à população que compõe a pesquisa, pois é possível que diferentes efeitos ocorram para indivíduos sem diagnóstico de depressão e com diferentes níveis de sintomas depressivos (leve, moderado e grave). Ainda, sugere-se que autores estejam atentos a proporção entre os sexos nos estudos, já que essa variável também pode afetar os resultados ${ }^{46}$.

\section{CONCLUSÃO}

Estudos a respeito da relação entre o eixo intestino-cérebro e sintomas depressivos (incluindo a DM) ainda estão em fase exploratória. Mais estudos experimentais randomizados, 
duplos-cegos ou triplos-cegos e placebo-controlados são necessários para que seja possível inferir relações de causalidade entre o uso de probióticos e o alívio de sintomas depressivos. Estudos futuros devem fundamentar as escolhas de instrumentos e seus pontos de corte, delimitar judiciosamente os critérios de inclusão e exclusão, e controlar fatores de confusão ao longo do estudo como dieta e atividade física, a fim de que os resultados sejam genuínos. Recomenda-se intervir utilizando somente uma espécie de bactéria e realizar follow-up. É possível que diferentes resultados sejam encontrados para intervenções realizadas com indivíduos saudáveis e com diferentes graus de severidade em sintomas depressivos (leve, moderado e grave), portanto estudos futuros devem explorar esse aspecto.

\section{CONTRIBUIÇÕES INDIVIDUAIS}

Flávia Bellesia Souzedo - Realizou buscas nas bases de dados, redigiu o artigo, realizou a análise dos dados e aprovou a versão final.

Lisiane Bizarro - Colaborou com a ideia do design utilizado, realizou revisão crítica do artigo em diferentes etapas e aprovou a versão final.

Ana Paula Almeida de Pereira - Colaborou com a ideia do design utilizado, orientou o trabalho como um todo, realizou revisão crítica do artigo em diferentes etapas e aprovou a versão final.

\section{CONFLITO DE INTERESSES}

As autoras declaram não haver conflito de interesses e suporte financeiro para o presente estudo.

\section{AGRADECIMENTOS}

As autoras declaram não haver agradecimentos a serem feitos.

\section{REFERÊNCIAS}

1. Moloney RD, Desbonnet L, Clarke G, Dinan TG, Cryan JF. The microbiome: Stress, health and disease. Mamm Genome. 2014;25(1-2):49-74.

2. Fond G, Boukouaci W, Chevalier G, Regnault A, Eberl G, Hamdani N, et al. The "psychomicrobiotic": Targeting microbiota in major psychiatric disorders: A systematic review. Pathol Biol (Paris). 2015;63(1):35-42.

3. Dinan TG, Cryan JF. Brain-gut-microbiota axis and mental health. Psychosom Med. 2017;79(8):920-6.

4. Biedermann $L$, Rogler $G$. The intestinal microbiota: its role in health and disease. Eur J Pediatr. 2015;174(2):151-67.

5. Foster JA, McVey Neufeld KA. Gut-brain axis: how the microbiome influences anxiety and depression. Trends Neurosci. 2013;36(5):305-12.
6. Slyepchenko A, Maes M, Jacka FN, Köhler CA, Barichello T, McIntyre RS, et al. Gut microbiota, bacterial translocation, and interactions with diet: pathophysiological links between major depressive disorder and non-communicable medical comorbidities. Psychother Psychosom. 2017;86(1):31-46.

7. Turnbaugh PJ, Ley RE, Hamady M, Fraser-Liggett CM, Knight R, Gordon JI. The human microbiome project. Nature. 2007;449(7164):804-10.

8. Alam R, Abdolmaleky HM, Zhou JR. Microbiome, inflammation, epigenetic alterations, and mental diseases. Am J Med Genet B Neuropsychiatr Genet. 2017;174(6):651-60.

9. Dinan TG, Cryan JF. Regulation of the stress response by the gut microbiota: implications for psychoneuroendocrinology. Psychoneuroendocrinology. 2012;37(9):1369-78.

10. Lach G, Schellekens H, Dinan TG, Cryan JF. Anxiety, depression, and the microbiome: a role for gut peptides. Neurotherapeutics. 2018;15(1):36-59

11. Dinan TG, Stanton C, Cryan JF. Psychobiotics: a novel class of psychotropic. Biol Psychiatry. 2013;74(10):720-6.

12. Cryan JF, O'Mahony SM. The microbiome-gut-brain axis: from bowel to behavior. Neurogastroenterol Motil. 2011;23(3):187-92.

13. Diaz Heijtz R, Wang S, Anuar F, Qian Y, Björkholm B, Samuelsson A, et al. Normal gut microbiota modulates brain development and behavior. Proc Natl Acad Sci U S A. 2011;108(7):3047-52.

14. Luczynski P, McVey Neufeld KA, Oriach CS, Clarke G, Dinan TG, et al. Growing up in a bubble: using germ-free animals to assess the influence of the gut microbiota on brain and behavior. Int J Neuropsychopharmacol. 2016;19(8):pyw020.

15. Neufeld KM, Kang N, Bienenstock J, Foster JA. Reduced anxiety-like behavior and central neurochemical change in germ-free mice. Neurogastroenterol Motil. 2011;23(3):255-64, e119.

16. Wallace CJK, Milev R. The effects of probiotics on depressive symptoms in humans: a systematic review. Ann Gen Psychiatry. 2017;16:14.

17. Desbonnet L, Garrett L, Clarke G, Kiely B, Cryan JF, Dinan TG. Effects of the probiotic Bifidobacterium infantis in the maternal separation model of depression. Neuroscience. 2010;170(4):1179-88

18. Guida F, Turco F, lannotta M, De Gregorio D, Palumbo I, Sarnelli G, et al. Antibioticinduced microbiota perturbation causes gut endocannabinoidome changes, hippocampal neuroglial reorganization and depression in mice. Brain Behav Immun. 2018;67:230-45.

19. Burokas A, Arboleya S, Moloney RD, Peterson VL, Murphy K, Clarke G, et al. Targeting the microbiota-gut-brain axis: prebiotics have anxiolytic and antidepressant-like effects and reverse the impact of chronic stress in mice. Biol Psychiatry. 2017;82(7):472-87.

20. Vrieze A, de Groot PF, Kootte RS, Knaapen M, van Nood E, Nieuwdorp M. Fecal transplant: a safe and sustainable clinical therapy for restoring intestinal microbial balance in human disease? Best Pract Res Clin Gastroenterol. 2013;27(1):127-37.

21. De Palma G, Lynch MD, Lu J, Dang VT, Deng Y, Jury J, et al. Transplantation of fecal microbiota from patients with irritable bowel syndrome alters gut function and behavior in recipient mice. Sci Transl Med. 2017;9(379):eaaf6397.

22. Evrensel A, Ceylan ME. The gut-brain axis: the missing link in depression. Clin Psychopharmacol Neurosci. 2015;13(3):239-44.

23. Mayer EA, Knight R, Mazmanian SK, Cryan JF, Tillisch K. Gut microbes and the brain: paradigm shift in neuroscience. J Neurosci. 2014;34(46):15490-6.

24. Szeligowski T, Yun AL, Lennox BR, Burnet PWJ. The Gut Microbiome and Schizophrenia: The Current State of the Field and Clinical Applications. Front Psychiatry. 2020;11:156.

25. Dash S, Clarke G, Berk M, Jacka FN. The gut microbiome and diet in psychiatry: focus on depression. Curr Opin Psychiatry. 2015;28(1):1-6.

26. Aizawa E, Tsuji H, Asahara T, Takahashi T, Teraishi T, Yoshida S, et al. Possible association of Bifidobacterium and Lactobacillus in the gut microbiota of patients with major depressive disorder. J Affect Disord. 2016;202:254-7.

27. Jiang H, Ling Z, Zhang Y, Mao H, Ma Z, Yin Y, et al. Altered fecal microbiota composition in patients with major depressive disorder. Brain Behav Immun. 2015;48:186-94.

28. Schachter J, Martel J, Lin CS, Chang CJ, Wu TR, Lu CC, et al. Effects of obesity on depression: a role for inflammation and the gut microbiota. Brain Behav Immun. 2018;69:1-8.

29. D'Mello C, Swain MG. Immune-to-brain communication pathways in inflammationassociated sickness and depression. In: Dantzer R, Capuron L. Inflammation-Associated Depression: Evidence, Mechanisms and Implications. Cham: Springer; 2016, p. 73-94. 
30. Waclawiková B, El Aidy S. Role of microbiota and tryptophan metabolites in the remote effect of intestinal inflammation on brain and depression. Pharmaceuticals (Basel). 2018;11(3):63.

31. Kurokawa S, Kishimoto T, Mizuno S, Masaoka T, Naganuma M, Liang KC, et al. The effect of fecal microbiota transplantation on psychiatric symptoms among patients with irritable bowel syndrome, functional diarrhea and functional constipation: an open-label observational study. Affect Disord. 2018;235:506-12.

32. Sudo N, Chida Y, Aiba Y, Sonoda J, Oyama N, Yu XN, et al. Postnatal microbial colonization programs the hypothalamic-pituitary-adrenal system for stress response in mice. J Physiol. 2004;558(Pt 1):263-75

33. Dinan TG, Stanton C, Cryan JF. Psychobiotics: a novel class of psychotropic. Biol Psychiatry. 2013;74(10):720-6.

34. Dinan TG, Cryan JF. Melancholic microbes: a link between gut microbiota and depression? Neurogastroenterol Motil. 2013;25(9):713-9.

35. Eisch AJ, Petrik D. Depression and hippocampal neurogenesis: a road to remission? Science. 2012;338(6103):72-5.

36. Desbonnet L, Garrett L, Clarke G, Kiely B, Cryan JF, Dinan TG. Effects of the probiotic Bifidobacterium infantis in the maternal separation model of depression. Neuroscience. 2010;170(4):1179-88.

37. Savignac HM, Kiely B, Dinan TG, Cryan JF. Bifidobacteria exert strain-specific effects on stress-related behavior and physiology in BALB/C mice. Neurogastroenterol Motil. 2014;26(11):1615-27.

38. Abildgaard A, Elfving B, Hokland M, Lund S, Wegener G. Probiotic treatment protects against the pro-depressant-like effect of high-fat diet in Flinders Sensitive Line rats. Brain Behav Immun. 2017;65:33-42.

39. Schachter J, Martel J, Lin CS, Chang CJ, Wu TR, Lu CC, et al. Effects of obesity on depression: a role for inflammation and the gut microbiota. rain Behav Immun. 2018;69:1-8.

40. American Psychiatric Association. DSM-5: Manual Diagnóstico e Estatístico de Transtornos Mentais. Porto Alegre: Artmed; 2014.

41. Koopman M, El Aidy S; MIDtrauma consortium. Depressed gut? The microbiota-dietinflammation trialogue in depression. Curr Opin Psychiatry. 2017;30(5):369-77.

42. Dash S, Clarke G, Berk M, Jacka FN. The gut microbiome and diet in psychiatry: focus on depression. Curr Opin Psychiatry. 2015;28(1):1-6.

43. Foster JA, McVey Neufeld KA. Gut-brain axis: how the microbiome influences anxiety and depression. Trends Neurosci. 2013;36(5):305-12.

44. Kennedy PJ, Cryan JF, Dinan TG, Clarke G. Kynurenine pathway metabolism and the microbiota-gut-brain axis. Neuropharmacology. 2017;112(Pt B):399-412.

45. Akkasheh G, Kashani-Poor Z, Tajabadi-Ebrahimi M, Jafari P, Akbari H, Taghizadeh M, et al. Clinical and metabolic response to probiotic administration in patients with major depressive disorder: a randomized, double-blind, placebo-controlled trial. Nutrition. 2016;32(3):315-20.

46. Steenbergen L, Sellaro R, van Hemert S, Bosch JA, Colzato LS. A randomized controlled trial to test the effect of multispecies probiotics on cognitive reactivity to sad mood. Brain Behav Immun. 2015;48:258-64.

47. Pinto-Sanchez MI, Hall GB, Ghajar K, Nardelli A, Bolino C, Lau JT, et al. Probiotic Bifidobacterium longum NCC3001 reduces depression scores and alters brain activity: a pilot study in patients with irritable bowel syndrome. Gastroenterology. 2017;153(2):448-59.e8.

48. Romijn AR, Rucklidge JJ, Kuijer RG, Frampton C. A double-blind, randomized, placebocontrolled trial of Lactobacillus helveticus and Bifidobacterium longum for the symptoms of depression. Aust N Z J Psychiatry. 2017;51(8):810-21.
49. Kazemi A, Noorbala AA, Azam K, Eskandari MH, Djafarian K. Effect of probiotic and prebiotic vs placebo on psychological outcomes in patients with major depressive disorder: A randomized clinical trial. Clinical Nutrition. 2019;38(2):522-8.

50. Majeed M, Nagabhushanam K, Arumugam S, Majeed S, Ali F. Bacillus coagulans MTCC 5856 for the management of major depression with irritable bowel syndrome: a randomised, double-blind, placebo controlled, multi-centre, pilot clinical study. Food Nutr. 2018;62.

51. Chahwan B, Kwan S, Isik A, van Hemert S, Burke C, Roberts L. Gut feelings: a randomised, triple-blind, placebo-controlled trial of probiotics for depressive symptoms. J Affect Disord. 2019;253:317-26.

52. Rudzki L, Ostrowska L, Pawlak D, Małus A, Pawlak K, Waszkiewicz N, et al. Probiotic Lactobacillus Plantarum 299v decreases kynurenine concentration and improves cognitive functions in patients with major depression: A double-blind, randomized, placebo controlled study. Psychoneuroendocrinology. 2019;100:213-22.

53. Freire MÁ, Figueiredo VD, Gomide A, Jansen K, Silva RD, Magalhães PVDS, et al. Escala Hamilton: estudo das características psicométricas em uma amostra do sul do Brasil. J Bras Psiquiatr. 2014;63(4):281-9.

54. Bagby RM, Ryder AG, Schuller DR, Marshall MB. The Hamilton Depression Rating Scale: has the gold standard become a lead weight? Am J Psychiatry. 2004;161(12):2163-77.

55. Montgomery SA, Asberg M. A new depression scale designed to be sensitive to change. Br J Psychiatry. 1979;134:382-9.

56. Iannuzzo RW, Jaeger J, Goldberg JF, Kafantaris V, Sublette ME. Development and reliability of the HAM-D/MADRS interview: an integrated depression symptom rating scale. Psychiatry Res. 2006;145(1):21-37.

57. Gorenstein C, Andrade LHSG. Inventário de depressão de Beck: propriedades psicométricas da versão em português. Rev Psiquiatr Clin. 1998;25(5):245-50.

58. Fantino B, Moore N. The self-reported Montgomery-Åsberg depression rating scale is a useful evaluative tool in major depressive disorder. BMC Psychiatry. 2009;9:26.

59. Kang SS, Jeraldo PR, Kurti A, Miller ME, Cook MD, Whitlock K, et al. Diet and exercise orthogonally alter the gut microbiome and reveal independent associations with anxiety and cognition. Mol Neurodegener. 2014;9:36.

60. Flint HJ, Duncan SH, Scott KP, Louis P. Links between diet, gut microbiota composition and gut metabolism. Proc Nutr Soc. 2015;74(1):13-22.

61. Cummings JH, Macfarlane GT. Gastrointestinal effects of prebiotics. Br J Nutr. 2002;87(\$2):S145-51.

62. Burokas A, Arboleya S, Moloney RD, Peterson VL, Murphy K, Clarke G, et al. Targeting the microbiota-gut-brain axis: prebiotics have anxiolytic and antidepressant-like effects and reverse the impact of chronic stress in mice. Biol Psychiatry. 2017;82(7):472-87.

63. Macedo D, Filho AJMC, Soares de Sousa CN, Quevedo J, Barichello T, Júnior HVN, et al. Antidepressants, antimicrobials or both? Gut microbiota dysbiosis in depression and possible implications of the antimicrobial effects of antidepressant drugs for antidepressant effectiveness. J Affect Disord. 2017;208:22-32.

64. Guida F, Turco F, lannotta M, De Gregorio D, Palumbo I, Sarnelli G, et al. Antibioticinduced microbiota perturbation causes gut endocannabinoidome changes, hippocampal neuroglial reorganization and depression in mice. Brain Behav Immun. 2018;67:230-45.

65. Kelly JR, Borre Y, O' Brien C, Patterson E, El Aidy S, Deane J, et al. Transferring the blues: depression-associated gut microbiota induces neurobehavioural changes in the rat. J Psychiatr Res. 2016;82:109-18. 